\title{
Un diagnóstico de situación de la empresa social en Espańa
}

\author{
A State Diagnostic of the Social Enterprise in Spain \\ Isadora SÁNCHEZ-Torné*, Macarena PÉREZ-SuÁREZ \\ Personal Docente e Investigador del Departamento de Economía Aplicada III \\ de la Universidad de Sevilla
}

\begin{abstract}
Resumen: El objetivo de la investigación fue identificar el estado actual del tejido empresarial social de España, así como detallar el perfil de la Empresa Social activa. En esta identificación se planteó una investigación inductiva mediante un análisis cuantitativo a partir de fuentes secundarias, en particular, de directorios públicos de Empresas Sociales localizadas en España. A partir de los cuales se elaboró una base de datos-directorio propio con varias variables comunes (tamaño, año de constitución, región, género) sobre las características de las empresas. Obtenidos los datos, se trataron en SPSS, en un análisis estadístico descriptivo e inferencial de una población de 123 empresas. Se determinaron varias características definitorias de las actuales Empresas Sociales de España, como por ejemplo la concentración territorial, dado que la mayoría se ubica en cuatro regiones con fuerte arraigo de Economía Social (Cataluña, Comunidad de Madrid, Comunidad Valenciana y País Vasco).
\end{abstract}

Palabras clave: Empresa Social, Empresas de Inserción, Centros Especiales de Empleo y Cooperativas de Iniciativa Social, España.

\begin{abstract}
The objective of the research was to identify the current state of the social business in Spain, as well as detailing the profile of the active Social Company. In this identification, an inductive investigation was proposed through a quantitative analysis from secondary sources, in particular, from public directories of Social Companies located in Spain, from which an own database-directory was configured with several common variables (size, year of incorporation, region, gender) about the characteristics of the companies. Obtained the data, they were treated in SPSS, in a descriptive and inferential statistical analysis of a population of 123 companies. In this way, several defining characteristics of the current Social Companies in Spain were determined, such as territorial concentration, since most are located in four regions with strong roots in the Social Economy (Cataluña, Comunidad de Madrid, Comunidad Valenciana y País Vasco).
\end{abstract}

Keywords: Social Enterprise, Insertion Companies, Special Employment Centers and Social Initiative Co-operatives, Spain.

Claves Econlit: L30 M10, P13.

* Correspondencia a/Corresponding author: Isadora Sánchez-Torné. Departamento de Economía Aplicada III de la Universidad de Sevilla - isanchez6@us.es - https://orcid.org/0000-0003-2749-2896

Cómo citar/How to cite: Sánchez-Torné, Isadora; Pérez-Suárez, Macarena (2020). «Un diagnóstico de situación de la empresa social en España», GIZAEKOA - Revista Vasca de Economía Social, 17, 7-34. (https://doi.org/10.1387/gizaekoa.22225).

Recibido: 09/10/2019; aceptado: 23/10/2019.

ISSN 1698-7446 - elSSN 2444-3107 / (C) 2020 UPV/EHU

(c) 


\section{Introducción}

La investigación se justifica a partir de varias premisas como que la Empresa Social es una realidad socioeconómica ascendente en los últimos años, especialmente en el período de crisis. En particular, Europa dispone de una desarrollada experiencia en este modelo organizacional que halla sus raíces en la Economía Social y el Tercer Sector, así, atiende a su promoción con iniciativas emblemáticas pro-Empresas Sociales. En último lugar, existen las evidencias de un tratamiento académico en auge y una conceptualización explícita.

A nivel territorial, se reconoce que hay mayor número de Empresas Sociales en Reino Unido, pues el emprendimiento es de tradición y el estado de bienestar está limitado desde el sector público; y en Francia, por su atención a la ayuda social. No obstante, en el sur de Europa hay un alto interés en impulsar estas iniciativas ${ }^{1}$ como demuestra que la Comisión Europea lanza la iniciativa Social Business Iniciative (SBI) ${ }^{2}$ y el Programa de la Unión Europea para el Empleo y la Innovación Social 2014-2020 (EaSI) ${ }^{3}$ a favor de un ecosistema europeo común de emprendimiento social. Con relación al concepto europeo de Empresa Social, es «de amplia tradición en Europa y desarrollado durante muchos años bajo diferentes formas jurídicas, al que se pueden incorporar elementos novedosos que están apareciendo en los últimos años en contextos de pobreza en países en desarrollo. En concreto, dentro de las nuevas propuestas de empresas comprometidas con el desarrollo humano» (Fisac-García, Moreno-Romero, Mataix y Palacios, 2011, p.14).

En España, se identifica un aumento reciente de Empresas Sociales, según datos publicados por la entidad Cáritas, estas entidades obtuvieron una facturación en su conjunto de 37 millones de euros con una inversión en acciones de inserción laboral de 27 millones de euros en $2016^{4}$. No obstante, el país no dispone de unas fuentes fiables sobre este sector formado por una variedad de modelos organizacionales. Si que parece haber consenso en que las empresas que tienen proyectos destinados y/o residentes en España están muy centradas en ayudar a colectivos en riesgo de exclu-

1 Pérez de Pablos, S. (2013). (Consultado 01.08.2019). https://elpais.com/sociedad/ 2013/02/24/actualidad/1361743315_132277.html

2 Comisión Europea. (Consultado 05.08.2019). https://ec.europa.eu/growth/sectors/socialeconomy/enterprises_en

3 Comisión Europea. (Consultado 05.08.2019). https://ec.europa.eu/social/main. jsp?catId=1081\&langId=es

${ }^{4}$ Cáritas Trabajo. (Consultado 01.08.2019). http://www.alfayomega.es/107937/caritascuenta-ya-con-49-empresas-de-economia-social-en-espana 
sión ${ }^{5}$. Mientras que, las compañías enfocadas a la ayuda en los países en desarrollo buscan cubrir necesidades básicas (la alimentación, la electricidad, el agua potable, la educación o la sanidad) ${ }^{6}$. Las tasas de emprendimiento global no siguen una tendencia uniforme, donde Espańa presenta una mínima tasa de actividad emprendedora social (un $0,51 \%$ frente al $4,25 \%$ de Islandia, por ejemplo) ${ }^{7}$ según el informe Global Entrepreneurship Monitor $2014^{8}$.

Caro-González, Pérez-Suárez y Sánchez-Torné (2019, p. 129) reconocen la vinculación entre la Empresa Social y la Economía Social, pues «desde el punto de vista práctico, absolutamente todas las organizaciones que se consideran parte integrante de la economía solidaria también forman parte inequívoca de la Economía Social. Lo mismo puede decirse de otros desarrollos teóricos como el de tercer sector de utilidad social». Específicamente, «la Comisión Europea no solo facilitó una descripción de las características de las empresas sociales, sino que afirmó expresamente que conforman una parte integrante de la Economía Social: "una Empresa Social, agente de la Economía Social, es una empresa cuyo principal objetivo es tener una incidencia social (...) (Comunicación de la Comisión Europea, Iniciativa en favor del emprendimiento social, COM/2011/0682 final de 25 de octubre de 2011)"” (MonzónCampos y Chaves-Ávila, 2012, pp. 21-24). Fajardo (2014, p. 79) puntualiza que la Empresa Social es un agente de la Economía Social que «funciona en el mercado proporcionando bienes y servicios de manera empresarial e innovadora y utiliza sus excedentes principalmente para fines sociales».

Tal como detallan Díaz-Foncea, Marcuello y Marcuello (2012, p. 181), «la Comisión Europea ha realizado una llamada para tener en cuenta a las empresas sociales como parte de la Economía Social y como uno de los actores que hacen posibles los objetivos marcados en la Estrategia 2020 de la Unión Europea». Lo que constata Nogales-Muriel (2017, p. 120) cuando afirma que «la mayoría de las empresas sociales europeas tienen sus raíces

5 Personas discapacitadas, personas enfermas mentales, personas ex drogodependientes, personas expresidiarias, mujeres maltratadas, personas paradas de larga duración o jóvenes con formación escasa.

6 Pérez de Pablos, S. (2013). (Consultado 01.08.2019). https://elpais.com/sociedad/ 2013/02/24/actualidad/1361743315_132277.html

7 APD. «Informe Forética 2015 sobre el estado de la Responsabilidad Social Empresarial en España». (Consultado 01.08.2019). https://www.apd.es/la-importancia-del-emprendimientosocial-espana/

8 Global Entrepreneurship Monitor. «Informe GEM España». (Consultado 01.08.2019). http://www.gem-spain.com/wp-content/uploads/2015/04/GEM_es_2014.pdf 
en la Economía Social, que reúne a todas aquellas organizaciones cuyo objetivo principal es servir a sus socios o una comunidad en lugar de buscar la maximización de la rentabilidad económica».

En el momento de desarrollar el concepto de Empresa Social, referir a la escuela europea, cuyo principal valedor es EMES. Este enfoque tiene un amplio uso dentro de la literatura académica (Díaz-Foncea y Marcuello, 2014). También cuenta con el reconocimiento de las instituciones, como señalan Monzón-Campos y Chaves-Ávila (2012, pp.26-27), pues «el enfoque EMES conforma el concepto de Empresa Social que ha prevalecido en los documentos de las instituciones de la Unión Europea» (Caro-González et al., 2019, p.131).

La definición de Empresa Social de EMES ${ }^{9}$ (European Research Network ${ }^{10}$ ) identifica once criterios agrupados en tres dimensiones: económica y emprendedora, social y gobernanza participativa (Defourny y Nyssens, 2014). Entendiendo a estas empresas como "aquellas organizaciones privadas no lucrativas que proporcionan bienes y servicios directamente relacionados con su objetivo explícito de beneficio a la comunidad» (Díaz-Foncea y Marcuello, 2014, p.146). «La ausencia de fin lucrativo es una protección frente a dos fenómenos negativos: la selección adversa y el riesgo moral» (Laville, 2015, p.94). Para Fisac-García et al. (2011, p.5), la organización «se apoya en dinámicas colectivas que incluyen diferentes tipos de grupos de interés en sus órganos de gobierno, tienen una fuerte autonomía y soportan riesgos económicos asociados a su actividad». Aunque reconocen que existe una ambigüedad en el concepto dada la diferencia de enfoques académicos, la falta de un marco teórico común, y la frecuencia con la que el término Empresa Social procede de la traducción de distintos términos no equivalentes (Fisac-García et al., 2011, p.4). A la definición, le complementan otros trabajos como el de Fisac-García y MorenoRomero (2015), pues identifica ocho elementos como las características que concretan a la Empresa Social, entre ellos están, el impacto social, la gobernanza, el ecosistema colaborativo y la orientación a la sostenibilidad económica.

${ }^{9}$ La red europea EMES (EMES es el acrónimo en francés de un gran amplio proyecto de investigación llevado a cabo sobre la "Emergencia de las Empresas Sociales en Europa» (1996-1999). Originalmente se refería al grupo de investigadores que desarrollaron este proyecto para la Dirección General de Investigación de la Comunidad Europea. El nombre se mantuvo en los sucesivos proyectos y en la actualidad, la red EMES está compuesta por nueve centros de investigación especializados en estos temas, así como otros investigadores particulares de toda Europa) — dedicada al estudio de las empresas sociales (Fisac-García et al., 2011, p. 5).

10 Red europea EMES. (Consultado 05.08.2019). https://emes.net/ 
La definición ya fue distinguida por Argudo (2011, p.180), al mostrar que las empresas sociales se caracterizan "por su finalidad social, su carácter no lucrativo y su gestión de carácter empresarial». Salinas y Rubio (2001, p.102) rotulan que «la acción de la Empresa Social genera cohesión social y promueve una acción basada en la solidaridad, pero al mismo tiempo se la desplaza de aquellos espacios más rentables, progresivamente capturados por las empresas lucrativas». Mientras que, Díaz-Foncea, Marcuello y Marcuello (2012, p.183) marcan que "la definición de Empresa Social requiere de una aproximación multidimensional en la que se conjugan elementos tradicionales de la empresa y de la Economía Social, que afectan especialmente al objeto social, la motivación económica y los modelos de gestión de este tipo de organizaciones». Finalmente, Solórzano-García, GuzmánAlfonso, Savall-Morera y Villajos-Girona (2018, p.160), matizan que las empresas sociales tienen como objetivo «la generación de valor social para los beneficiarios de su misión social y su fuente de ingresos principal es la vía comercial, dependiendo del mercado para su sostenibilidad financiera, de forma que las actividades comerciales se entienden como medios para obtener sus fines».

La academia aborda la variedad de condiciones de aparición y consolidación de la Empresa Social en disímiles contextos, la diversidad de modelos organizacionales existentes, y la evolución y los factores socioculturales para su progreso. Si bien en el escenario nacional la tendencia se inclina a un tratamiento conceptual en detrimento del tratamiento empírico. En particular, se revelan «diferentes modelos de empresas sociales en función de su procedencia: existen diferencias entre la tradición europea y de la tradición anglosajona» (Díaz-Foncea y Marcuello, 2014, p.145). Además, de tratar el contraste y la similitud con la empresa capitalista, y sus formas jurídicas adoptadas (Spear, Cornforth y Aiken, 2009; Díaz-Foncea, Marcuello y Marcuello, 2012).

La principal aportación de valor a la comunidad científica de este trabajo es discutir algunos tópicos que se utilizan acríticamente en relación con el fenómeno estudiado. Además de, generar conocimiento para la comunidad (la Administración Pública, las personas vinculadas al emprendimiento social, las personas representantes de la Economía Social, los sindicatos, etc.), pues se cree contribuir tanto a la sostenibilidad futura de este tipo de entidades, como a su integración real en la Economía Social.

Determinado el contexto, el objetivo de la investigación es identificar el actual tejido empresarial social de España. Sin duda, plantear un estudio exploratorio inicial que se estructura en cuatro partes, la primera dedicada a limitar la revisión de la literatura. Seguidamente, se explica la metodología para analizar los datos. En tercer lugar, se exponen los resultados 
obtenidos, y, por último, las conclusiones halladas que dan respuestas al objetivo fijado. En definitiva, esta investigación viene a sumar valor en la creación de conocimiento y en el tratamiento de una realidad específica y singular, que al mismo tiempo que proporciona información a diversas partes involucradas del ámbito académico nacional, insta a trabajar en exploraciones científicas más ambiciosas.

\section{Revisión de la Literatura}

El origen de la Empresa Social de España reside en la Economía Social, el Tercer Sector de acción social y la Economía Solidaria (NogalesMuriel, 2017), hallándose los primeros ejemplos de Empresa Social en el siglo Xv (Solórzano-García et al., 2018). Tal como detalla Nogales-Muriel (2017, p.130), «en España, la Empresa Social no aparece en los últimos años de modo aislado, en el vacío. De hecho, tal desarrollo se debe en gran medida al excepcional caldo de cultivo que supone la presencia de la Economía Social, el tercer sector de acción social y la economía solidaria». Ciertamente, Solórzano-García et al. (2018, p.155) especifican que «se pueden identificar ejemplos de empresas sociales en nuestro país desde el siglo XV».

Las investigaciones desarrolladas en España sobre las empresas sociales son limitadas y de tendencia teórica, generando oportunidades científicas en aras de estudios empíricos. Se pueden identificar tres líneas de trabajos: el proceso de las organizaciones no lucrativas de acción social a las empresas sociales (Salinas y Rubio, 2001; Argudo, 2011; Fisac-García et al., 2011); la identificación de los modelos organizativos de empresas sociales en España (Díaz-Foncea y Marcuello, 2014; Nogales-Muriel, 2017); y la delimitación socioeconómica de la Empresa Social (Solórzano-García et al., 2018). Tres líneas de trabajo teóricas que hallan su excepción en los trabajos empíricos sobre las empresas de inserción (EI) (Díaz-Foncea, Marcuello y Marcuello, 2012; Paniagua, 2013). Además, es remarcado por Nogales-Muriel (2017, p.125), cuando recoge que «según cifras oficiales analizadas a 2016, el número estimado total de empresas sociales en España durante 2014 asciende a 8.410 de las cuales 656 son empresas de inserción (EI)». En consecuencia, no se hallan evidencias específicas de los otros tipos de entidades sociales en el ámbito territorial de España.

A nivel teórico, se identifican tres tipos principales de entidades sociales, las llamadas Work Integration Social Enterprises (WISEs) (Davies, Haugh y Chambers, 2018): las Empresas de Inserción (EI), los Centros Especiales de Empleo (CEE) y las Cooperativas de Iniciativa Social (CIS) 
(Díaz-Foncea y Marcuello, 2014; Díaz-Foncea et al., 2017; Nogales-Muriel, 2017; Solórzano-García et al., 2018). A las que habría que sumar, algunas asociaciones y fundaciones (Argudo, 2011), la Sociedad Cooperativa de Impulso Empresarial y las Sociedades Cooperativas de Servicios Públicos de la Ley 14/2011 de Cooperativas Andaluzas (Nogales-Muriel, 2017). Unas organizaciones "principalmente vinculadas con el modelo europeo de Empresa Social, como deja vislumbrar la relación existente entre las características de estas entidades y los criterios incluidos en el modelo» (Díaz-Foncea y Marcuello, 2014, p.161). Por tanto, conocer las características de estas entidades es el objetivo sustancial del presente trabajo. En este rasgo, Solórzano-García et al. (2018, p.165) afirman que su «análisis bibliográfico no fue suficiente para identificar las dimensiones que permitieran construir un marco teórico coherente para establecer una tipología de empresas sociales españolas, pero permitió identificar un conjunto de cuestiones que reflejan los principios que rigen la constitución y el comportamiento de las empresas sociales». Desde la corriente de pensamiento, Díaz-Foncea, Marcuello y Marcuello (2012, p.184) afirman que «las empresas sociales en Espańa han seguido una trayectoria similar al resto de los países de la Unión Europea y predomina el modelo vinculado a la Economía Social. Asimismo, en esta trayectoria se comparten elementos de reflexión como la necesidad de innovar nuevas formas de actuación para adecuarse a las necesidades de las personas (...) En el caso de España se concretaron en las Empresas de Inserción y los Centros Especiales de Empleo. Por otro lado, también a finales del siglo xx se crearon las Cooperativas de Iniciativa Social».

En relación con las Cooperativas de Iniciativa Social (CIS), Argudo (2011, p.183) detalla que "pueden configurarse, al menos teóricamente y por previsión legal, como especialidad de cualquier tipo de cooperativa dentro de las clasificaciones que se contemplan legislativamente» (art. 106 de la Ley 27/1999 de Cooperativas). La especialidad consiste en que han de calificarse como de ánimo social siendo obligatorio que el objeto social contemple alguna de las actividades del Art.106, constar en los Registros de Cooperativas y reunir una serie de requisitos reflejados en los estatutos sociales («cooperativa sin ánimo de lucro») (Argudo, 2011, p.184). En España, «el término «cooperativa social» no es recogido expresamente en la legislación, por lo que se trata de una denominación "de conveniencia» con la intención de definir a un conjunto concreto de cooperativas. De este modo, las cooperativas sociales españolas vienen reguladas mediante dos tipos de figuras jurídicas: las cooperativas de integración social y las cooperativas de iniciativa social» (Burgo, 2014, p.77). Todas ellas se describen por «su fin mutualista (atender las necesidades y aspiraciones de sus socias y sus socios), pues cumplen una función de in- 
terés social destacable» (Fajardo, 2014, pp.1-6). Según Solórzano-García et al., (2018, p.167), "estas entidades cumplen todas las características del sector de la Economía Social (ya que las cooperativas constituyen la forma jurídica más representativa) con la característica adicional de estar dedicadas a servir el interés general de la sociedad. En España, éstas surgen al final del siglo xx con el propósito de crear dentro del marco nacional de Economía Social un modelo organizativo similar al de las cooperativas sociales italianas».

Los trabajos empíricos nacionales sobre las Empresas de Inserción (EI) se hallan a lo largo del quindenio reciente (Puig, 1998; Álvarez, 1999; Coque y Pérez, 2000; Rojo, 2000; López-Aranguren, 2002; FAEDEI, 2003; Millán, 2006; García y Esteve, 2007; Marcuello, 2007; Quintão, 2007; Marcuello, Bellostas y Marcuello, 2008; Marcuello y Marcuello, 2010; Melián, Campos y Sanchis, 2011; Paniagua, 2013). En la práctica, las EI tienden a adoptar en España una diversidad de figuras jurídicas, predominando las asociaciones y fundaciones frente a las sociedades limitadas. Lo cual sucede, fundamentalmente, a partir de las reducciones fiscales y la mayor valoración que reciben por parte de la comunidad. Tal como ha sido demostrado, su desarrollo incide directamente en el nivel de cohesión social y solidaridad de la sociedad ${ }^{11}$, siendo imprescindible la participación del mayor número posible de agentes socioeconómicos (Calvo,2005, pp.115-118). De estas razones puede derivarse la recomendación de replantear el modelo de inserción dada por Retolaza, San José y Pruñonosa (2013:100).

La realidad de los Centros Especiales de Empleo (CEE) en la economía española es abordada desde el ámbito empresarial social (Monzón-Campos y Herrero-Montagud, 2016; López, Maside y Torrelles, 2019) y normativo (Moratalla, 2016).

En síntesis, tal como señalan Solórzano-García et al. (2018, p.160), «a nivel nacional, establecer una clasificación de empresas sociales es aún una tarea pendiente, sobre todo, teniendo en cuenta cómo pueden variar las realidades objeto de estudio de un territorio a otro y los distintos enfoques». No obstante, se hallan algunos intentos de clasificación de las Empresas Sociales en Espańa, como el dado por Villajos y Sanchis (2013) y el proyecto internacional ICSEM (Díaz-Foncea et al., 2017). Estos limitados intentos pueden derivar de que hay ciertos factores que han ralentizado el desarrollo de la Empresa Social en España: «el legado histórico de la distinción entre Economía Social, economía solidaria y tercer sector de acción

11 Ejemplo es, tal como recogen Aretxabala y Caro (2013, p.180), que en Euskadi la inmensa mayoría de las EI cuenten con personas inmigrantes internacionales. 
social que ha resultado en una separación de facto entre las organizaciones que se engloban en uno u otro sector; la fuerte implantación y el alto nivel de institucionalización de la Economía Social y del tercer sector, así como gradualmente la economía solidaria; y, la aparición de modelos de emprendimiento social que enfatizan la iniciativa individual y que se apoyan en recursos de origen eminentemente privados, lo que los ha aislado de la tradición espańola de grupos organizados» de ciudadanía (Nogales-Muriel, 2017, p.132).

Finalmente, sobre el estado de la literatura de la Empresas Sociales de España, se puede decir que: la investigación es limitada y de tendencia teórica, generando oportunidades científicas para estudios empíricos venideros. Así como, hay que destacar las investigaciones sobre la identificación de los modelos organizativos, donde la mayoría enumera tres tipos principales: Empresas de Inserción, Centros Especiales de Empleo y Cooperativas de Iniciativa Social. Tratándose de organizaciones con características propias, pues «enfrentan una serie de desafíos específicos en su accionar en el mercado, entre ellos el de su sostenibilidad, ya que, si quieren mantenerse en el tiempo y así provocar cambios sociales significativos y duraderos - con todos los beneficios que ello conlleva para los territorios en los que actúan-, deben generar no sólo valor social, sino que también valor económico» (Torres, 2017, p.63).

A partir de ahí, y considerando estas investigaciones previas, se formulan dos hipótesis:

$-\mathrm{H}_{1}$ : Las Empresas Sociales españolas se crean en periodo de crisis económica siendo significativo el despunte de los últimos años.

$-\mathrm{H}_{2}$ : Las Empresas Sociales españolas ostentan unas características propias.

\section{Metodología}

En esta investigación se analizó a las Empresas Sociales españolas, para ello, en primer lugar, fue preciso elaborar un directorio propio. Este se nutrió de tres Websites de Empresas Sociales (Social Enterprises) de España: Ashoka; Red emprendeverde-Fundación Biodiversidad y Redcreactiva. Lo cual permitió encontrar una población objeto de estudio de 123 empresas sociales. Esta labor de recopilación de información se llevó a cabo en el mes de marzo de 2019.

A continuación, para conocer las características de estas empresas se aplicó un tratamiento estadístico de la información hallada, se plateó un estudio agregado de las 123 corporaciones. Para ello se empleó Statistical 
Software IBM SPSS (23v), con el que se realizó un análisis descriptivo e inferencial.

El análisis descriptivo tuvo como objetivo identificar el actual Perfil de Empresa Social activa y agrupar el año de creación de la empresa por el mayor número de respuestas obtenidas para establecer el ciclo de vida empresarial (Padilla, Fabricio y Jiménez, 2018). Inicialmente, el ciclo de vida se clasificó de la siguiente forma: nacimiento (1 año de vida), crecimiento ( 2 a 7 ańos), madurez (8 a 16 años) y madurez avanzada (16 años en adelante). Posteriormente, se procedió a realizar una agrupación dual: grupo de nacimiento y crecimiento y grupo de madurez y madurez avanzada.

El análisis de inferencias consistió en la aplicación de la prueba $Z$ para contrastar proporciones a un nivel de confianza del $95 \%$ y el error del $5 \%$, con el objetivo estudiar si existen diferencias significativas en el perfil de los dos grupos indicados anteriormente.

En último lugar, hay que apuntar que en los cálculos no se contabiliza las respuestas $\mathrm{Ns} / \mathrm{Nc}$, y que se han tratado diversas variables como los servicios prestados, el impacto social en la comunidad, el tipo de empresa, la localización (Comunidad Autónoma), el volumen de facturación, el número de personas empleadas y el número de personas socias. 


\section{Resultados}

\subsection{El perfil de la Empresa Social activa en España}

\subsubsection{Servicios PRESTAdos}

La mayoría de las empresas sociales se dedica a los servicios de Educación $(16,26 \%)$ y los servicios de Programación, consultoría y otras actividades relacionadas con la Informática $(13,01 \%)$.

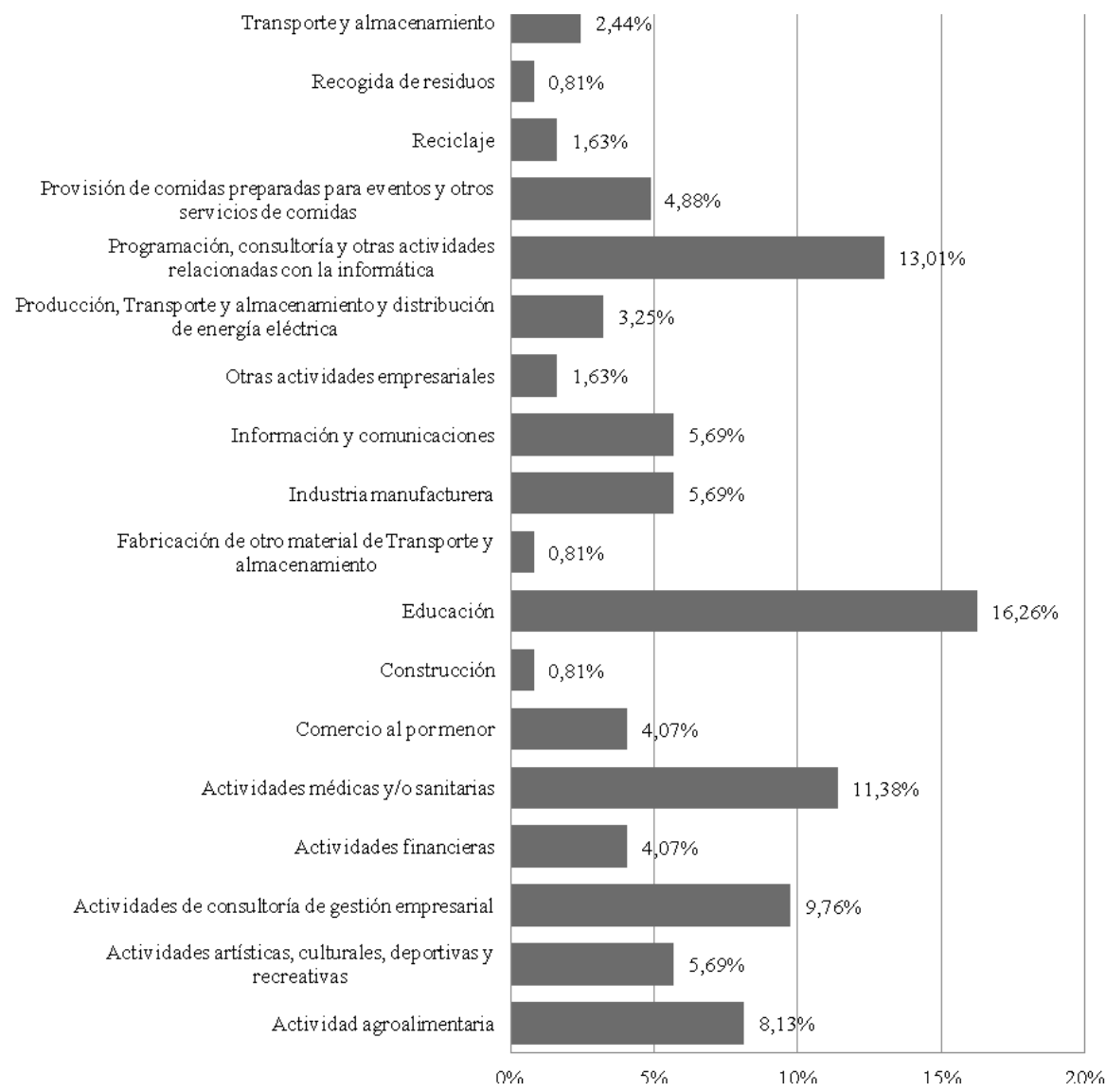

Fuente. Elaboración propia a partir de Ashoka; Red emprendeverde-Fundación Biodiversidad y Redcreactiva (marzo 2019).

\section{Gráfico 1}

Servicios prestados por las Empresas Sociales de España 


\subsubsection{SeCTOR DE PRODUCCIÓN}

Tal como avanzaba el gráfico anterior, las Empresas Sociales de España conforman las actividades económicas del Sector Terciario (87\%).

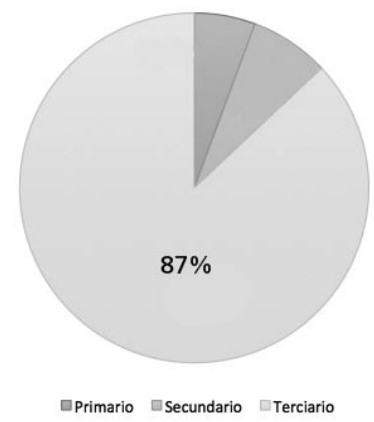

Fuente. Elaboración propia a partir de Ashoka; Red emprendeverde-Fundación Biodiversidad y Redcreactiva (marzo 2019).

\section{Gráfico 2}

Sector de Producción de las Empresas Sociales de España

\subsubsection{Tipo de Impacto Social}

El tipo de impacto social en la comunidad responde a una significativa mayoría dedicada la Inclusión social y laboral (47,15\%). Seguida de manera prudente por el Desarrollo rural y ambiental (16,26\%) y la Mejora del aprendizaje $(15,45 \%)$.
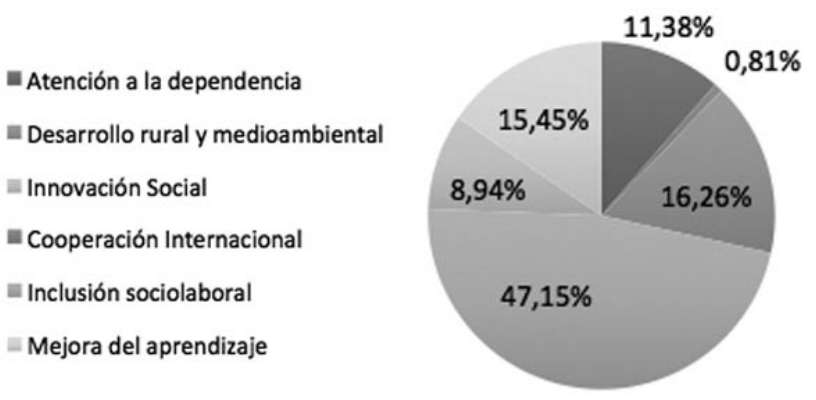

Fuente. Elaboración propia a partir de Ashoka; Red emprendeverde-Fundación Biodiversidad y Redcreactiva (marzo 2019).

\section{Gráfico 3}

Tipo de Impacto Social en la comunidad de las Empresas Sociales de España 


\subsubsection{TIPO DE EMPRESA}

La forma jurídica de la Empresa Social de Espańa es una Sociedad Limitada (57.38\%), mayoritariamente, o bien una Fundación (25.41\%). Un resultado que presenta oposición a lo señalado por Aretxabala y Caro (2013), que le otorgaba en España una mayoría a las fundaciones frente a las sociedades limitadas.

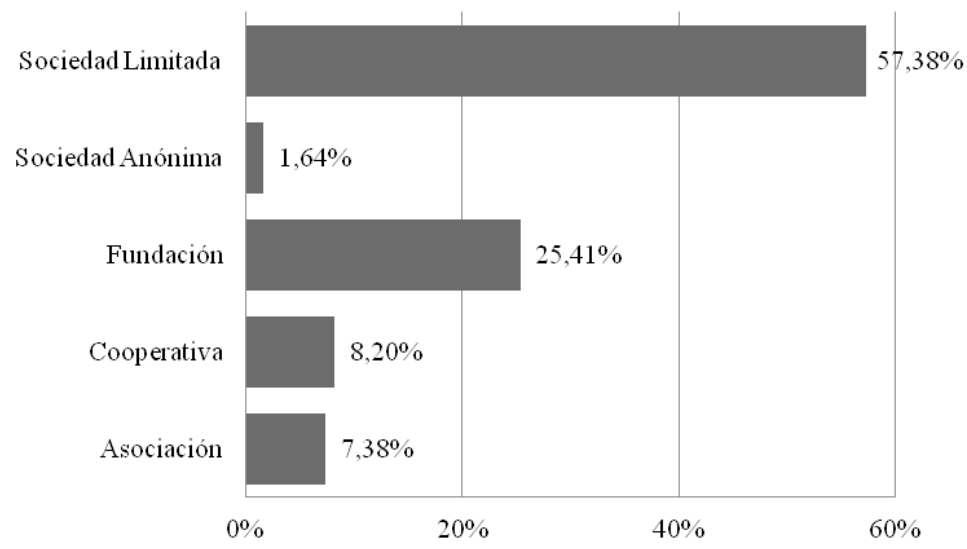

Fuente. Elaboración propia a partir de Ashoka; Red emprendeverde-Fundación Biodiversidad y Redcreactiva (marzo 2019).

\section{Gráfico 4}

Forma Jurídica de las Empresas Sociales de España

\subsubsection{LOCALIZACión}

La Empresa Social de España reside, en su mayoría, en la comunidad autónoma de Cataluña (30.08\%) y en la Comunidad de Madrid (28.46\%). También se ubican en la Comunidad Valenciana y el País Vasco. De lo cual se puede intuir la influencia de la Economía Social, pues citadas regiones son sus pioneras, a excepción de Andalucía que no se muestra significativa en estas averiguaciones y sí en el ránking nacional de Economía Social.

De igual modo, los datos obtenidos señalan consonancia con el Informe Global Entrepreneurship Monitor (2014) ${ }^{12}$, pues pone de relieve que Madrid es una región de relevante porcentaje de población adulta involucrada en actividades de emprendimiento social. Por el contrario, La Rioja se encuentra a la cola por el volumen de personas emprendedoras sociales.

12 Global Entrepreneurship Monitor. «Informe GEM España». (Consultado 01.08.2019). http://www.gem-spain.com/wp-content/uploads/2015/04/GEM_es_2014.pdf 
Tabla 1

Localización de las Empresas Sociales de España

\begin{tabular}{lcc}
\hline \multicolumn{1}{c}{ Comunidad Autónoma } & Frecuencia & Porcentaje \\
\hline Andalucía & 6 & $4,88 \%$ \\
Aragón & 4 & $3,25 \%$ \\
Castilla y León & 6 & $4,88 \%$ \\
Castilla-La Mancha & 1 & $0,81 \%$ \\
Cataluña & 37 & $30,08 \%$ \\
Comunidad de Madrid & 35 & $28,46 \%$ \\
Comunidad de Murcia & 1 & $0,81 \%$ \\
Comunidad Valenciana & 12 & $9,76 \%$ \\
Extremadura & 1 & $0,81 \%$ \\
Galicia & 6 & $4,88 \%$ \\
Islas Baleares & 1 & $0,81 \%$ \\
La Rioja & 1 & $0,81 \%$ \\
Navarra & 1 & $0,81 \%$ \\
País Vasco & 11 & $8,94 \%$ \\
\hline
\end{tabular}

Fuente. Elaboración propia a partir de Ashoka; Red emprendeverde-Fundación Biodiversidad y Redcreactiva (marzo 2019).

\subsubsection{Ciclo de vida empresarial}

$\mathrm{Al}$ analizar el ciclo de vida empresarial, se halla una mayoría en las empresas de 8 a 16 años de vida, pudiendo ser denominadas empresas maduras. Por otra parte, el $57.94 \%$ de las Empresas Sociales españolas se han creado durante el último período de crisis económica (2008-2016).

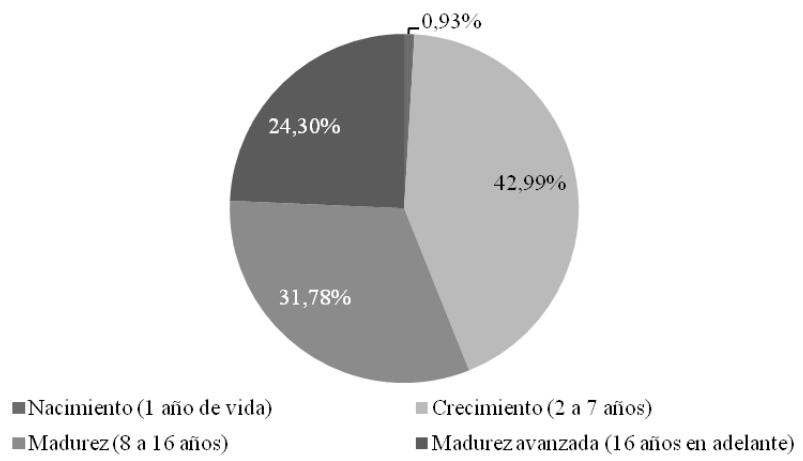

Fuente. Elaboración propia a partir de Ashoka; Red emprendeverde-Fundación Biodiversidad y Redcreactiva (marzo 2019).

\section{Gráfico 5}

Ciclo de vida empresarial de las Empresas Sociales de España 


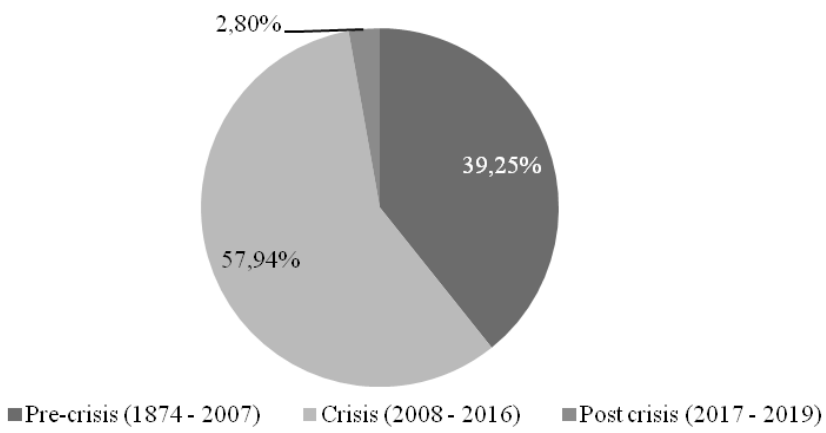

Fuente. Elaboración propia a partir de Ashoka; Red emprendeverdeFundación Biodiversidad y Redcreactiva (marzo 2019).

\section{Gráfico 6}

Periodo de Constitución de las Empresas Sociales de España

\subsubsection{Volumen de FACTURACIÓN}

El $61.91 \%$ de las Empresas Sociales de España factura de 50.001 a $900.000 €$.

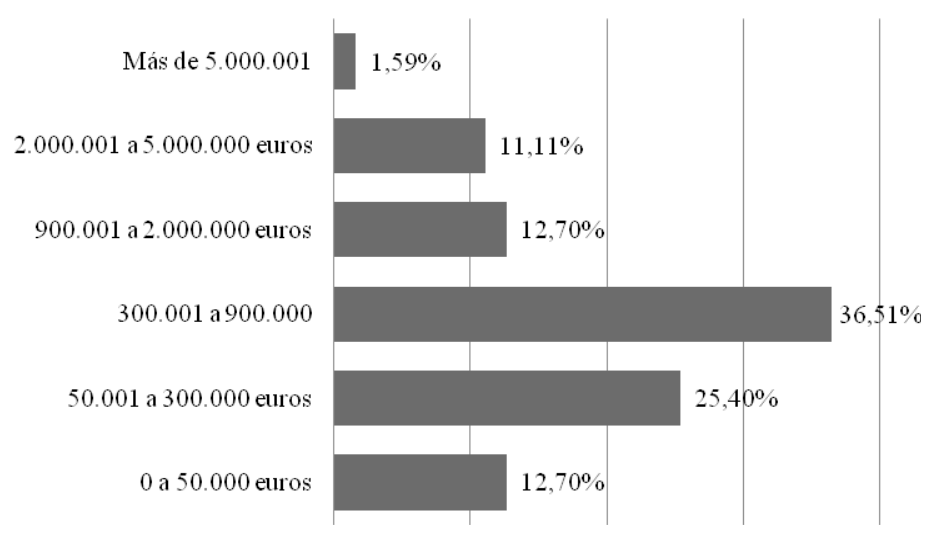

Fuente. Elaboración propia a partir de Ashoka; Red emprendeverdeFundación Biodiversidad y Redcreactiva (marzo 2019).

\section{Gráfico 7}

Volumen de facturación de las Empresas Sociales de España 


\subsubsection{NÚMERO DE PERSONAS EMPLEADAS}

Al detallar la unión de la variable número de personas empleadas y género, se observa como el 20\% de las Empresas Sociales en España tiene entre el $61 \%$ y el $99 \%$ de trabajadoras mujeres en su plantilla. En otras palabras, el 10,91\% de las Empresas Sociales en España tiene entre el 1\% a $20 \%$ de mujeres en su plantilla, mientras que el $7,27 \%$ tiene entre $1 \%$ a $20 \%$ de hombres en su equipo. De igual modo, se podría puntualizar que el 34,55\% de las Empresas Sociales en España tiene entre 61\% a 99\% de hombres en su plantilla, mientras que el $20 \%$ de las empresas sociales posee entre el $61 \%$ a $99 \%$ de mujeres en el organigrama.

\section{Tabla 2}

Género de la plantilla empleada en las Empresas Sociales de España

\begin{tabular}{rrr}
\hline Porcentaje de personas trabajadoras & Hombres & Mujeres \\
\hline 0\% personas trabajadoras & $7,27 \%$ & $7,27 \%$ \\
$1 \%$ a 20\% personas trabajadoras & $7,27 \%$ & $10,91 \%$ \\
$21 \%$ a 40\% personas trabajadoras & $14,55 \%$ & $25,45 \%$ \\
$41 \%$ a $60 \%$ personas trabajadoras & $29,09 \%$ & $29,09 \%$ \\
$61 \%$ a $99 \%$ personas trabajadoras & $34,55 \%$ & $20,00 \%$ \\
$100 \%$ personas trabajadoras & $7,27 \%$ & $7,27 \%$ \\
\hline
\end{tabular}

Fuente. Elaboración propia a partir de Ashoka; Red emprendeverde-Fundación Biodiversidad y Redcreactiva (marzo 2019).

\subsubsection{NÚMERO DE PERSONAS SOCIAS}

$\mathrm{Al}$ especificar la unión de la variable número de personas socias y género, se observa que el $52.56 \%$ de las Empresas Sociales en Espańa tiene una participación femenina del $0 \%$. En otras palabras, el 28,21\% de las Empresas Sociales en Espańa tienen un 100\% de personas socias femeninas.

\section{Tabla 3}

Género de las personas socias en las Empresas Sociales de España

\begin{tabular}{crr}
\hline \multicolumn{1}{r}{ Porcentaje de personas socias } & Hombres & Mujeres \\
\hline $0 \%$ & $28,21 \%$ & $52,56 \%$ \\
$1 \mathrm{a} 49 \%$ & $2,56 \%$ & $5,13 \%$ \\
$50 \%$ a $99 \%$ & $16,67 \%$ & $14,10 \%$ \\
$100 \%$ & $52,56 \%$ & $28,21 \%$ \\
\hline
\end{tabular}

Fuente. Elaboración propia a partir de Ashoka; Red emprendeverde-Fundación Biodiversidad y Redcreactiva (marzo 2019). 
Este resultado se verifica con lo señalado por el Informe Global Entrepreneurship Monitor (GEM) España 2017-18, sobre que la Tasa de Actividad Emprendedora (TEA) presenta una participación masculina relativamente mayor que la femenina $(55,1 \%$ frente $44,9 \%)$; no obstante, la brecha entre hombres y mujeres a la hora de emprender disminuye de forma continua desde $2012^{13}$.

\subsubsection{E-COMMERCE}

El 67.26\% de las Empresas Sociales en España no dispone de comercio electrónico.

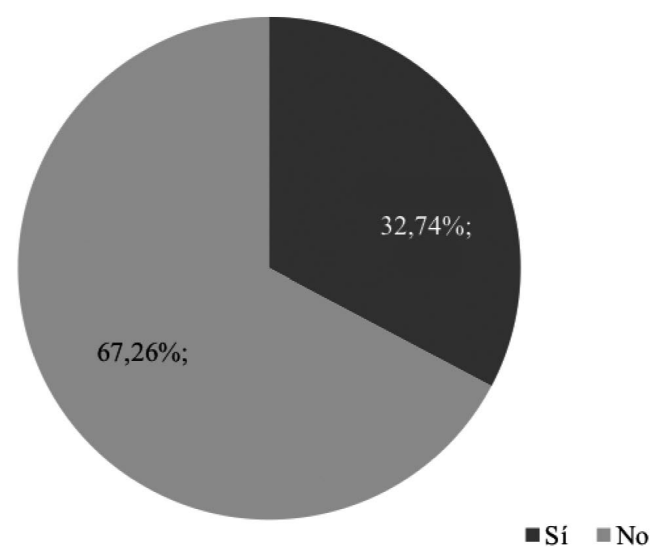

Fuente. Elaboración propia a partir de Ashoka; Red emprendeverde-

Fundación Biodiversidad y Redcreactiva (marzo 2019).

\section{Gráfico 8}

E-commerce en las Empresas Sociales de España

Según las averiguaciones halladas, se puede detallar un arquetipo de la actual Empresa Social de España, tal como marca la Tabla 4.

13 Global Entrepreneurship Monitor. «Informe GEM España 2017-18». (Consultado 01.08.2019). http://www.gem-spain.com/wp-content/uploads/2018/04/Informe-GEM2017-18.pdf 
Tabla 4

El perfil de la Empresa Social (ES) activa en España

\begin{tabular}{l|l}
\hline \multicolumn{1}{c|}{ Variables } & \multicolumn{1}{c}{ Derivaciones principales } \\
\hline Servicios prestados & Educación $(16,26 \%)$ \\
\hline Sector productivo & Terciario $(87 \%)$ \\
\hline Tipo de impacto social & Inclusión sociolaboral (47,15\%) \\
\hline Forma jurídica & Sociedad Limitada $(57,38 \%)$ \\
\hline Comunidad autónoma & Cataluńa $(30,08 \%)$ \\
\hline Facturación & 300.001 a $900.000 €(36.51 \%)$ \\
\hline Tamaño & $\begin{array}{l}\text { Microempresa }(1 \text { a } 9 \text { personas trabajadoras) } \\
(54.55 \%)\end{array}$ \\
\hline Personas trabajadoras según género & $\begin{array}{l}\text { Más Hombres que Mujeres (34,55\% ES } \\
\text { tiene entre } 61 \% \text { a } 99 \% \text { de Hombres). }\end{array}$ \\
\hline Personas socias según género & $\begin{array}{l}\text { Más Hombres que Mujeres }(52,56 \% \text { ES } \\
\text { tiene un } 0 \% \text { de socias). }\end{array}$ \\
\hline E-commerce & Sin E-commerce $(67,26 \%)$ \\
\hline
\end{tabular}

Fuente. Elaboración propia a partir de Ashoka; Red emprendeverde-Fundación Biodiversidad y Redcreactiva (marzo 2019).

\subsection{El perfil de la Empresa Social activa en España según el ciclo de vida empresarial}

En base a las derivaciones anteriores sobre el ciclo de vida empresarial de las Empresas Sociales, se decide profundizar en él. Así, se hallan dos grupos: el grupo de Nacimiento y Crecimiento y el grupo de Madurez y Madurez Avanzada. En el reconocimiento de los dos grupos, se encuentran diferencias significativas en cuatro variables $\left({ }^{*}\right)$ : Servicios prestados, Tipo de impacto social, Forma Jurídica y Facturación. Tal como muestra la Tabla 5 estas derivaciones plantean la importancia de seguir indagando en la materia.

Las empresas que se encuentran en su fase de nacimiento y crecimiento se caracterizan por actividades del sector de la educación $(23,40 \%)$, programación y consultoría $(14,89 \%)$, con una vocación de mejora del aprendizaje $(25,53 \%)$ e inserción sociolaboral $(31,91 \%)$, empleando mayoritariamente la forma jurídica de sociedad limitada $(76,60 \%)$, con un volumen 
de facturación entre 0 a 50.000 euros (25\%). En contraposición, las fundaciones aumentan su peso $(33,33 \%)$ cuando pasan a la etapa de madurez y madurez avanzada y el intervalo de facturación ( 0 a 50.000 euros en 5,26\%). Entre los servicios prestados, destaca su porcentaje dedicado a las actividades agroalimentaria (13,33\%).

Por último, se observa que en las empresas con mayor edad aumenta el porcentaje de mujeres trabajadoras y socias.

\section{Tabla 5}

El perfil de la Empresa Social activa en España según el ciclo de vida empresarial

\begin{tabular}{|c|c|c|c|}
\hline & & $\begin{array}{c}\text { Nacimiento } \\
y \\
\text { crecimiento }\end{array}$ & $\begin{array}{c}\text { Madurez y } \\
\text { Madurez } \\
\text { avanzada }\end{array}$ \\
\hline \multirow{18}{*}{ Servicios } & Actividad agroalimentaria* & $2,13 \% *$ & $13,33 \% *$ \\
\hline & $\begin{array}{l}\text { Actividades artísticas, culturales, deportivas } \\
\text { y recreativas }\end{array}$ & $2,13 \%$ & $5,00 \%$ \\
\hline & $\begin{array}{l}\text { Actividades de consultoría de gestión em- } \\
\text { presarial }\end{array}$ & $6,38 \%$ & $11,67 \%$ \\
\hline & Actividades financieras & $6,38 \%$ & $1,67 \%$ \\
\hline & Actividades médicas y/o sanitarias & $10,64 \%$ & $15,00 \%$ \\
\hline & Comercio al por menor & $4,26 \%$ & $5,00 \%$ \\
\hline & Construcción & $2,13 \%$ & $0,00 \%$ \\
\hline & Educación & $23,40 \%$ & $11,67 \%$ \\
\hline & $\begin{array}{l}\text { Fabricación de otro material de Transporte } \\
\text { y almacenamiento }\end{array}$ & $0,00 \%$ & $1,67 \%$ \\
\hline & Industria manufacturera & $4,26 \%$ & $8,33 \%$ \\
\hline & Información y comunicaciones & $6,38 \%$ & $6,67 \%$ \\
\hline & Otras actividades empresariales & $0,00 \%$ & $1,67 \%$ \\
\hline & $\begin{array}{l}\text { Producción, Transporte y almacenamiento } \\
\text { y distribución de energía eléctrica }\end{array}$ & $6,38 \%$ & $0,00 \%$ \\
\hline & $\begin{array}{l}\text { Programación, consultoría y otras activida- } \\
\text { des relacionadas con la informática }\end{array}$ & $14,89 \%$ & $8,33 \%$ \\
\hline & $\begin{array}{l}\text { Provisión de comidas preparadas para even- } \\
\text { tos y otros servicios de comidas }\end{array}$ & $8,51 \%$ & $3,33 \%$ \\
\hline & Reciclaje & $2,13 \%$ & $1,67 \%$ \\
\hline & Recogida de residuos & $0,00 \%$ & $1,67 \%$ \\
\hline & Transporte y almacenamiento & $0,00 \%$ & $3,33 \%$ \\
\hline
\end{tabular}




\begin{tabular}{|c|c|c|c|}
\hline & & $\begin{array}{c}\text { Nacimiento } \\
y \\
\text { crecimiento }\end{array}$ & $\begin{array}{c}\text { Madurez y } \\
\text { Madurez } \\
\text { avanzada }\end{array}$ \\
\hline \multirow{3}{*}{$\begin{array}{c}\text { Sector } \\
\text { Productivo }\end{array}$} & Primario & $2,13 \%$ & $10,00 \%$ \\
\hline & Secundario & $6,38 \%$ & $10,00 \%$ \\
\hline & Terciario & $91,49 \%$ & $80,00 \%$ \\
\hline \multirow{6}{*}{$\begin{array}{c}\text { Tipo de } \\
\text { impacto social }\end{array}$} & Atención a la dependencia & $10,64 \%$ & $10,00 \%$ \\
\hline & Cooperación Internacional & $0,00 \%$ & $1,67 \%$ \\
\hline & Desarrollo rural y medioambiental & $19,15 \%$ & $13,33 \%$ \\
\hline & Inclusión sociolaboral* & $31,91 \% *$ & $58,33 \% *$ \\
\hline & Innovación Social & $12,77 \%$ & $6,67 \%$ \\
\hline & Mejora del aprendizaje* & $25,53 \% *$ & $10,00 \% *$ \\
\hline \multirow{5}{*}{$\begin{array}{l}\text { Forma } \\
\text { jurídica }\end{array}$} & Asociaciones & $4,26 \%$ & $10,00 \%$ \\
\hline & Sociedad Limitada* & $76,60 \% *$ & $41,67 \% *$ \\
\hline & Sociedad Anónima & $2,13 \%$ & $1,67 \%$ \\
\hline & Fundaciones* & $12,77 \% *$ & $33,33 \% *$ \\
\hline & Cooperativas & $4,26 \%$ & $13,33 \%$ \\
\hline \multirow{13}{*}{ Localización } & Andalucía & $2,13 \%$ & $8,33 \%$ \\
\hline & Aragón & $6,38 \%$ & $1,67 \%$ \\
\hline & Castilla y León & $8,51 \%$ & $3,33 \%$ \\
\hline & Castilla-La Mancha & $0,00 \%$ & $1,67 \%$ \\
\hline & Cataluña & $25,53 \%$ & $36,67 \%$ \\
\hline & Comunidad de Madrid & $31,91 \%$ & $20,00 \%$ \\
\hline & Comunidad de Murcia & $2,13 \%$ & $0,00 \%$ \\
\hline & Comunidad Valenciana & $8,51 \%$ & $8,33 \%$ \\
\hline & Galicia & $2,13 \%$ & $6,67 \%$ \\
\hline & Islas Baleares & $0,00 \%$ & $1,67 \%$ \\
\hline & La Rioja & $2,13 \%$ & $0,00 \%$ \\
\hline & Navarra & $2,13 \%$ & $0,00 \%$ \\
\hline & País Vasco & $8,51 \%$ & $11,67 \%$ \\
\hline
\end{tabular}




\begin{tabular}{|c|c|c|c|}
\hline & & $\begin{array}{c}\text { Nacimiento } \\
y \\
\text { crecimiento }\end{array}$ & $\begin{array}{c}\text { Madurez y } \\
\text { Madurez } \\
\text { avanzada }\end{array}$ \\
\hline \multirow{6}{*}{$\begin{array}{l}\text { Volumen de } \\
\text { facturación }\end{array}$} & 0 a 50.000 euros* & $25,00 \% *$ & $5,26 \% *$ \\
\hline & 50.001 a 300.000 euros & $25,00 \%$ & $26,32 \%$ \\
\hline & 300.001 a 900.000 & $35,00 \%$ & $34,21 \%$ \\
\hline & 900.001 a 2.000 .000 euros & $10,00 \%$ & $15,79 \%$ \\
\hline & 2.000.001 a 5.000.000 euros & $5,00 \%$ & $15,79 \%$ \\
\hline & Más de 5.000 .001 & $0,00 \%$ & $2,63 \%$ \\
\hline \multirow{2}{*}{$\begin{array}{c}\text { Porcentaje } \\
\text { de personas } \\
\text { empleadas que } \\
\text { son mujeres }\end{array}$} & Menos del $50 \%$ empleados mujeres & $64,00 \%$ & $57,69 \%$ \\
\hline & $50 \%$ o más empleados mujeres & $36,00 \%$ & $42,31 \%$ \\
\hline \multirow{2}{*}{$\begin{array}{c}\text { Porcentaje } \\
\text { de personas } \\
\text { socias que son } \\
\text { mujeres }\end{array}$} & Menos del 50\% mujeres & $66,67 \%$ & $52,50 \%$ \\
\hline & $50 \%$ o más mujeres & $33,33 \%$ & $47,50 \%$ \\
\hline \multirow{2}{*}{ E-commerce } & Sí & $34,09 \%$ & $32,14 \%$ \\
\hline & No & $65,91 \%$ & $67,86 \%$ \\
\hline
\end{tabular}

* p-level <0,05.

Fuente. Elaboración propia a partir de Ashoka; Red emprendeverde-Fundación Biodiversidad y Redcreactiva (marzo 2019). 


\section{Conclusiones}

El objetivo de la investigación se logra al conocer el perfil vigente de la Empresa Social de España. Conforme a ello, se pueden enunciar varias premisas principales:

\section{Tabla 6}

Validación de las hipótesis de la investigación

\begin{tabular}{|c|c|c|}
\hline Hipótesis & Premisas averiguadas & Derivación \\
\hline $\begin{array}{l}\text { Las Empresas Sociales } \\
\text { españolas se crean en } \\
\text { periodo de crisis eco- } \\
\text { nómica siendo signifi- } \\
\text { cativo el despunte de } \\
\text { los últimos años. }\end{array}$ & $\begin{array}{l}\text { El } 57,94 \% \text { de las Empresas Sociales } \\
\text { españolas se incorpora en el último } \\
\text { periodo de crisis económica (2008- } \\
2016) ; \\
\text { Las Cooperativas de Iniciativa Social } \\
\text { inscritas se crearon entre finales del si- } \\
\text { glo xx y principios del siglo xxI. }\end{array}$ & Se sostiene \\
\hline $\begin{array}{l}\text { Las Empresas Socia- } \\
\text { les españolas osten- } \\
\text { tan unas características } \\
\text { propias. }\end{array}$ & $\begin{array}{l}\text { Las Empresas Sociales españolas son } \\
\text { Sociedades Limitadas }(57,38 \%) \text { que } \\
\text { se localizan en Cataluña }(30.08 \%) \\
\text { (además de en la Comunidad de Ma- } \\
\text { drid, Comunidad Valenciana y el País } \\
\text { Vasco). Las Empresas Sociales españo- } \\
\text { las constituyen un tejido empresarial } \\
\text { PYME que presenta como tipo de im- } \\
\text { pacto social la inclusión sociolaboral } \\
(47.15 \%) \text {. }\end{array}$ & Se sostiene \\
\hline
\end{tabular}

Fuente. Elaboración propia.

Además, en relación con las principales derivaciones halladas se pueden exponer cuáles han sido las premisas de partida y como se comprueban en la investigación (Tabla 7). 


\section{Tabla 7}

Principales comprobaciones de la investigación

\begin{tabular}{|c|c|}
\hline Premisa de partida según la Literatura & Demostración de la investigación \\
\hline $\begin{array}{l}\text { La Empresa Social es una realidad so- } \\
\text { cioeconómica ascendente en tiempos de } \\
\text { crisis. }\end{array}$ & $\begin{array}{l}\text { El } 57,94 \% \text { Empresas Sociales espańolas } \\
\text { activas se han constituido en el último } \\
\text { periodo de crisis económica. }\end{array}$ \\
\hline $\begin{array}{l}\text { Se identifican tres tipos principales de } \\
\text { entidades sociales: las Empresas de In- } \\
\text { serción (EI), los Centros Especiales de } \\
\text { Empleo (CEE) y las Cooperativas de } \\
\text { Iniciativa Social (CIS) (Díaz-Foncea y } \\
\text { Marcuello, 2014; Nogales-Muriel,2017; } \\
\text { Solórzano-García et al., 2018). }\end{array}$ & $\begin{array}{l}\text { Las Empresas Sociales españolas acti- } \\
\text { vas son Sociedades Limitadas }(57,38 \%) \text {; } \\
\text { Fundaciones }(25,41 \%) \text {; y Cooperativas } \\
\text { de Iniciativa Social }(8,20 \%) \text {. }\end{array}$ \\
\hline $\begin{array}{l}\text { «A nivel nacional, establecer una clasifi- } \\
\text { cación de empresas sociales es aún una } \\
\text { tarea pendiente, sobre todo, teniendo en } \\
\text { cuenta cómo pueden variar las realidades } \\
\text { objeto de estudio de un territorio a otro» } \\
\text { (Solórzano-García et al., 2018, p.160). }\end{array}$ & $\begin{array}{l}\text { Las Empresas Sociales españolas activas } \\
\text { se localizan en cuatro regiones principa- } \\
\text { les: Cataluña, Comunidad de Madrid, } \\
\text { Comunidad Valenciana y País Vasco. }\end{array}$ \\
\hline $\begin{array}{l}\text { "A finales del siglo xx se crearon las } \\
\text { Cooperativas de Iniciativa Social» (Díaz- } \\
\text { Foncea, Marcuello y Marcuello, 2012, } \\
\text { p.184; Solórzano-García et al., 2018, } \\
\text { p.167). }\end{array}$ & $\begin{array}{l}\text { Las Cooperativas de Iniciativa Social re- } \\
\text { gistradas se crearon entre finales del si- } \\
\text { glo xx y principios del siglo xxI. }\end{array}$ \\
\hline $\begin{array}{l}\text { «Una modelización de la Empresa So- } \\
\text { cial en el país en base a los sectores de } \\
\text { actividad, llegando a unos resultados } \\
\text { que no eran del todo concluyentes ... } \\
\text { (Díaz-Foncea et al., 2017) (...) No fue } \\
\text { suficiente para identificar las dimensio- } \\
\text { nes que permitieran construir un marco } \\
\text { teórico coherente para establecer una ti- } \\
\text { pología de empresas sociales españolas» } \\
\text { (Solórzano-García et al., 2018, p.160, } \\
\text { p.165). }\end{array}$ & $\begin{array}{l}\text { Se plantea una investigación explora- } \\
\text { toria inicial. Siendo una prueba piloto, } \\
\text { pues queda por comprender la relación } \\
\text { existente entre las características de estas } \\
\text { entidades y los criterios del modelo; y la } \\
\text { relación entre tamańo empresas sociales } \\
\text { (PYME) y el apoyo financiación pública. }\end{array}$ \\
\hline
\end{tabular}

Fuente. Elaboración propia. 
En consecuencia, se hallan limitadas evidencias empíricas sobre los tipos de entidades sociales a nivel nacional. Al mismo tiempo, esto supone una oportunidad científica. Se coincide con lo señalado por SolórzanoGarcía et al. (2018, p.160), «se puede encontrar en la literatura algunos intentos de clasificación (Villajos y Sanchis, 2013). En este sentido destaca la realización de una primera aproximación dentro del marco del proyecto internacional ICSEM (Díaz-Foncea et al., 2017)».

Por otra parte, en esta investigación se reconocen limitaciones en la literatura consultada y la metodología (el alcance temporal y las fuentes de datos). La subsanación en futuras investigaciones puede pasar por: profundizar la revisión de la literatura a partir de su extensión y el detalle del ciclo de vida de una empresa; indagar la tendencia del tipo de empresa; abordar una temporalidad más amplia a partir de recabar datos de varios años de las empresas sociales e incrementar los métodos de captación de datos, las variables observadas y las técnicas cuantitativas tratadas. Así como, se podría contemplar medir el impacto social mediante la tasa de recuperación de inversiones sociales — Social Return on Investment (SROI)—, pues este índice indica la eficacia y la eficiencia de las inversiones. Aunque tiene la carencia de trabajar con elementos tangibles y no con los intangibles $^{14}$ (Díaz-Foncea, Marcuello y Marcuello, 2012, p.187). No obstante, deben producirse significativos cambios en la estructuración del proceso de financiación de los emprendimientos sociales, pues España dispone de una sólida Economía Social, pero adolece de sistemas confiables, verificables y comparables de medición de su impacto ambiental y social. En síntesis, el país se encuentra en un momento favorable en materia de inversiones de impacto, en el establecimiento de mecanismos de medición estandarizados desde las políticas públicas ${ }^{15}$.

De igual modo, se podría plantear ciertas investigaciones pormenorizadas de las empresas sociales españolas activas, pues «académicamente es interesante analizar experiencias reales en el ámbito de las cooperativas de iniciativa social» (Burgo, 2014, p.78). También, se podría comprobar la relación de las empresas sociales con los Objetivos de Desarrollo Sostenible 2030 (ODS), pues son capaces de solucionar los problemas vinculados a los ODS.

Con relación a las implicaciones de la investigación, se genera conocimiento y éste se suma a las indagaciones testadas hasta el momento. De acuerdo con Díaz-Foncea y Marcuello (2014, p.159), «el proceso de crecimiento a largo plazo de las empresas sociales es un tema reciente en este

14 Pérez de Pablos, S. (2013). (Consultado 01.08.2019). https://elpais.com/ sociedad/2013/02/24/actualidad/1361743315_132277.html

15 Martín, J.M. (2019). (Consultado 30.08.2019). http://agendapublica.elpais.com/siqueremos-financiar-los-ods-hay-que-mirar-al-sector-privado/ 
ámbito de investigación». Finalmente, tal como señalan Solórzano-García et al. (2018, p.176), «a pesar de las particularidades que puedan existir, el caso español no es una excepción en el ámbito internacional de la realidad de la Empresa Social». En cualquier caso, se coincide con Nogales-Muriel (2017, p.122) en que «lo más importante de la Empresa Social no son los contornos precisos de su definición, sí es necesaria una noción común compartida que capture su valor ańadido, sobre todo a la hora de poner en marcha políticas públicas y acciones tanto ciudadanas como en el sector empresarial que impulsen su desarrollo».

\section{Bibliografía}

Álvarez Vega, M.I. (1999). Las Empresas de inserción social como nueva forma de organización empresarial. Especial referencia a su régimen jurídico. CIRIECEspaña, Revista de Economía Pública, Social y Cooperativa, 31, 47-84.

Aretxabala M.E. \& Caro, A. (2013). Las empresas de inserción vascas y sus entidades promotoras como ejemplo de buenas prácticas en la integración sociolaboral del colectivo de inmigrantes más vulnerable. Revista Vasca de Economía Social, 10, 149-188.

Argudo Périz, J.L. (2011). Las cooperativas sin ánimo de lucro: ¿vuelta a los orígenes o respuesta a nuevas necesidades sociales? Revista Vasca de Economía Social, 3, 179-201.

Burgo García, U. (2014). El movimiento cooperativo de las ikastolas: su revisión conceptual desde la perspectiva de las empresas sociales. Revista Vasca de Economía Social, 10, 71-96.

Calvo Vérgez, J.C. (2005). En torno al régimen jurídico de las Empresas de Inserción Social: Cuestiones conflictivas. Revista Vasca de Economia Social, 1, 85-118.

Caro-González, F.J., Pérez-Suárez, M., \& Sánchez-Torné, I. (2019). Características de las Empresas Sociales Periodísticas. CIRIEC-España, Revista de Economia Pública, Social y Cooperativa, 96, 121-154. https://doi.org/10.7203/ CIRIEC-E.96.12723

Coque, J. \& Pérez, E. (2000). Cómo gestionar la creación de empresas de inserción social: una perspectiva de participación dinámica, en Parras, M. (ed.) Inteligencia empresarial. La gestión del conocimiento en la empresa, 2 (pp. 333-43). Jaén: Instituto de Estudios Giennenses.

Davies, I.A., Haugh, H., \& Chambers, L. (2018). Barriers to social enterprise growth. Journal of Small Business Management, 1-21. https://doi.org/10.1111/ jsbm.12429

Defourny, J., \& Nyssens, M. (2014). Social co-operatives: When social Enterprises meet the co-operative tradition. Journal of Entrepreneurial and Organizatio- 
nal Diversity, 2(2), 11-33. Disponible en https://papers.ssrn.com/sol3/papers. cfm?abstract_id=2437884

Díaz-Foncea, M. et al. (2017). Social Enterprise in Spain: A Diversity of Roots and a Proposal of Models. ICSEM Working Papers, 29. Disponible en https:// zaguan.unizar.es/record/70184/

Díaz-Foncea, M., \& Marcuello, C. (2014). Las empresas sociales en Espańa: concepto y características. Revista Vasca de Economía Social, 8, 143-164.

Díaz-Foncea, M., Marcuello, C., \& Marcuello, C. (2012). Empresas sociales y evaluación del impacto social. CIRIEC-España, Revista de Economía Pública, Social y Cooperativa, 75, 181-198. Disponible en https:/www.redalyc.org/ pdf/174/17425798010.pdf

FAEDEI (2003). Identificación y diagnóstico integral de las Empresas de Inserción en España. Madrid: Editorial Popular.

Fajardo García, G. (2014). El concepto legal de Economía Social y la Empresa Social. Revista Vasca de Economía Social, 8, 63-84.

Fisac-García, R., \& Moreno-Romero, A.M. (2015). La Empresa Social: marco conceptual, contexto e información. Documentos AECA, Serie Responsabilidad Social Corporativa, 9, 1-94. Disponible en http://www.aeca1.org/ revistaeca/revista112/112.pdf

Fisac-García, R., Moreno-Romero, A.M., Mataix, C., \& Palacios, M. (2011). La Empresa Social: revisión de conceptos y modelo para el análisis organizativo. Revista Española del Tercer Sector, 41-66. Disponible en http://oa.upm. es/12404/

García, A. \& Esteve, M. (2007). Las Empresas de Inserción en España: normativa y características. CIRIEC-España, Revista de Economía Pública, Social y Cooperativa, 59, 153-78.

Laville, J.L. (2015). Asociarse para el bien común: Tercer Sector, Economia Social y Economía Solidaria. Barcelona: Icaria.

López, M.C., Maside, J. M., \& Torrelles, J. (2019). Análisis económico y social de los centros especiales de empleo: un estudio en Galicia. REVESCO: Revista de Estudios Cooperativos, 132, 169-194. https://doi.org/10.5209/reve.64305

López-Aranguren, L.M. (2002). Las empresas de inserción en España: un marco de aprendizaje para la inserción laboral. Madrid: Consejo Económico y Social.

Marcuello Servós, C. (2007). Empresas de inserción: puentes de inclusión en el mercado laboral. CIRIEC-España, Revista de Economia Pública, Social y Cooperativa, 59, 5-6.

Marcuello, C. \& Marcuello, C. (2010). Las Empresas de inserción, en Mozón, E. \& Monzón-Campos, J.L. (eds.), La Economía Social en España en el año 2008. Ámbito, magnitudes, actividades y tendencias (pp. 417-33). CIRIECEspaña, Valencia.

Marcuello, C., Bellostas, A. \& Marcuello, CH. (2008). Informe sobre las Empresas de Inserción en España, Valencia: CIRIEC-España. 
Melián, A., Campos, V., \& Sanchis, J.R. (2011). Emprendimiento social y empresas de inserción en Espańa. Aplicación del método Delphi para la determinación del perfil del emprendedor y las empresas sociales creadas por emprendedores. REVESCO: Revista de Estudios Cooperativos, 106, 150-172.

Millán, J.C. (2006). Empresas de Inserción. Santiago de Compostela: Instituto Gallego de Iniciativas Sociales y Sanitarias.

Monzón-Campos, J.L., \& Herrero-Montagud, M. (2016). Identificación y análisis de las características identitarias de la empresa social europea: aplicación a la realidad de los Centros Especiales de Empleo de la economía española. CIRIEC-España, Revista de Economía Pública, Social y Cooperativa, 87, 295326.

Monzón-Campos, J., \& Chaves-Ávila, R. (2012). Evolución reciente de la Economía Social en la Unión Europea. Bélgica: CIRIEC-International. CES/ CSS/12/2016/23406. Disponible en https://www.eesc.europa.eu/sites/default/ files/files/qe-04-17-875-es-n.pdf

Moratalla, P. (2016). Centros Especiales de Empleo. CIRIEC-España, Revista Jurídica de Economía Social y Cooperativa, 29, 235-273. Disponible en http:// ciriec-revistajuridica.es/wp-content/uploads/029-010.pdf

Nogales-Muriel, R. (2017). La Empresa Social en Europa y España: evolución, relevancia y desafíos. Revista Tercer Sector, 117-140. Disponible en http:// accioncontraelhambre.org:8080/sites/default/files/documents/tsector_35_1. pdf\#page $=117$

Padilla, M.P., Fabricio, L. \& Jiménez, W.R. (2018). La dinámica empresarial y el emprendimiento, factores determinantes para el desarrollo del ciclo de vida de las pymes. Revista Publicando, 5(15), 308-325.

Paniagua Zurera, M. (2013). Las empresas de inserción sociolaboral y las cláusulas sociales en la contratación pública: dos instrumentos necesarios para la innovación social hacia el desarrollo. REVESCO: Revista de Estudios Cooperativos, $112,176-212$.

Puig i Olle, A. (1998). Las Empresas de Inserción. El papel de las fundaciones y las asociaciones en la creación de las empresas. REVESCO: Revista de Estudios Cooperativos, 65, 69-71.

Quintão, C. (2007). Empresas de Inserción y Empresas Sociales en Europa. CIRIEC-España, Revista de Economía Pública, Social y Cooperativa, 59, 5-6.

Retolaza, J.L., San José, L., \& Pruñonosa, J.T. (2013). Las empresas de inserción en España: ¿una oportunidad perdida? Revista Vasca de Economía Social, 10, 97-123.

Rojo Giménez, C. (2000). Empresas de Inserción en Castilla y León. CIRIEC-España, Revista de Economía Pública, Social y Cooperativa, 36, 97-115.

Salinas, F., \& Rubio, M.J. (2001). Tendencias en la evolución de las organizaciones no lucrativas hacia la Empresa Social. CIRIEC-España, Revista de Economía Pública, Social y Cooperativa, 37, 79-116. Disponible en https://www.redalyc. org/pdf/174/17403705.pdf 
Solórzano-García, M., Guzmán-Alfonso, C., Savall-Morera, T., \& Villajos-Girona, E. (2018). La identidad de la Empresa Social en España: análisis desde cuatro realidades socioeconómicas. CIRIEC-España, Revista de Economía Pública, Social y Cooperativa, 92, 155-182. https://doi.org/10.7203/CIRIECE.92.9236

Spear, R., Cornforth, C., \& Aiken, M. (2009). The governance challenges of social enterprises: evidence from a UK empirical study. Annals of Public and Cooperative Economics, 80(2), 247-273. https://doi.org/10.1111/j.1467-8292. 2009.00386.x

Torres Ortega, J.A. (2017). Ecosistemas para el emprendimiento: Características del concepto y su aplicación a la Empresa Social. Revista Vasca de Economía Social, 14, 61-76. DOI: 10.1387/reves.18004

Villajos, E. \& Sanchis, J.R. (2013). Las empresas sociales y el management social. Revista Vasca de Economia Social, 10, 189-216. 